\title{
Las Lógicas del Trabajo Moral en Telemedicina: Disputas en Torno a la Legitimidad de Políticas de Salud Digital
}

\author{
The Logics of Moral Work in Telemedicine: \\ Disputes on the Legitimacy of Digital Health \\ Policies
}

\section{A Lógica do Trabalho Moral na Telemedicina: Disputas Sobre a Legitimidade das Políticas de Saúde Digital}

\author{
Fernando Valenzuela \\ ORCID ID: 0000-0002-7987-0150 \\ Universidad Andres Bello, Chile \\ Nairbis Sibrian \\ ORCID ID: 0000-0001-8008-5080 \\ Universidad Santo Tomás, Chile
}

Autor referente: fernando.valenzuela@unab.cl

Historia editorial Recibido: 20/03/2019 Aceptado: 03/09/2019

\section{RESUMEN}

A partir del estudio de un programa de teleneurología implementado en Biobío, Chile, este artículo analiza las lógicas que articulan el trabajo moral de la medicina a distancia. Desde un enfoque pragmático informado por la semiótica material, la teoría de la subjetivación y la sociología de la moralidad, este artículo analiza tres lógicas que dan sentido a prácticas de valuación del programa de teleneurología. Ellas son desplegadas por actores que desempeñan roles distintos, generando puntos de tensión y encauzando la constante evolución de los entramados sociomateriales en que se realiza el cuidado de salud. Una lógica cívico industrial fundamenta discursos públicos que destacan la reducción de las listas de espera de especialidad como prueba de valor del programa de telemedicina. Desde una 
lógica de la seguridad, en tanto, personal médico prioriza la adopción de estándares que, orientados a controlar los riesgos implicados en la realización de medicina a distancia, son tenidos como válidos por una comunidad profesional. Por su parte, adoptando una lógica del cuidado, pacientes y médicos generales enfatizan el despliegue de prácticas orientadas a atender la situación particular que experimenta cada paciente. Se propone que reconocer las lógicas que distintos actores puedan adoptar para otorgar valor a las prácticas de cuidado de salud puede favorecer la comprensión, anticipación y resolución de las tensiones que emergen con la implementación de tecnologías de salud digital y telemedicina.

Palabras clave: Evaluación de la tecnología; política de la salud; salud digital; telemedicina

\section{ABSTRACT}

Based on the study of a teleneurology program implemented in Biobío, Chile, this article analyses the logics that articulate the moral work of medicine at a distance. From a pragmatic approach informed by material semiotics, the theory of subjectivisation and the sociology of morality, this article analyses three logics that give meaning to practices of valuation of the teleneurology program. These are deployed by actors who play different roles, generating points of tension, and channeling the constant evolution of the socio-material networks in which health care is performed. A civic industrial logic underpins public discourses that highlight the reduction of waiting lists as proof of the value of the telemedicine program. From a safety logic, meanwhile, medical staff prioritizes the adoption of standards that, aimed at controlling the risks involved in the realisation of medicine remotely, are held as valid by a professional community. For their part, adopting logic of care, patients and general practitioners emphasize the deployment of practices aimed at attending the particular situation experienced by each patient. It is proposed that recognizing the logics that different actors may adopt when giving value to health care practices can promote understanding, anticipating and solving the tensions that arise with the implementation of digital health technologies and telemedicine.

Keywords: Technology assessment; health policy; digital health; telemedicine

\section{RESUMO}

Com base no estudo de um programa de teleneurologia implementado em Biobío, Chile, este artigo analisa as lógicas que articulam o trabalho moral da medicina à distância. A partir de uma abordagem pragmática informada pela semiótica material, a teoria da subjetivação e a sociologia da moralidade, este artigo analisa três lógicas que dão sentido às práticas de valorização do programa de teleneurologia. Eles são implantados por atores que desempenham diferentes papéis, gerando pontos de estresse e canalizando a constante evolução dos tecidos sociomateriais nos quais os cuidados de saúde são realizados. Uma lógica cívica industrial 
apoia discursos públicos que destacam o declínio das listas de espera de especialidade como prova de valor do programa de telemedicina. A partir de uma lógica de segurança, entretanto, a equipe médica prioriza a adoção de padrões que, visando controlar os riscos envolvidos na realização da medicina remotamente, são mantidos como válidos por uma comunidade profissional. Por sua vez, adotar uma lógica de cuidado, pacientes e clínicos gerais enfatizam a implantação de práticas voltadas ao atendimento da situação particular vivenciada por cada paciente. Propõe-se que o reconhecimento das lógicas que diferentes atores podem adotar para valorizar as práticas de saúde pode promover a compreensão, antecipação e resolução das tensões que surgem com a implementação das tecnologias de saúde digital e telemedicina.

Palavras-chave: Avaliação da tecnologia; política de saúde; saúde digital; telemedicina

o que encontramos al llegar a la Unidad de Teleprocesos del Hospital Las Higueras de Talcahuano, Chile, en junio de 2017, no coincidía del todo con lo que esperábamos. Esta unidad tuvo sus orígenes en 2008, cuando el cardiólogo Francisco Albornoz inició un proyecto piloto para hacer frente a la escasez de especialistas en los servicios de salud de la macro red de Biobío (Carvajal, González, \& Pacheco, 2013). Con los años se había convertido en un ejemplo de implementación exitosa de tecnologías de telemedicina en el servicio público de salud del país, siendo reconocida principalmente por su impacto en la reducción de listas de espera de especialidad. Habiendo iniciado con prestaciones de cardiología, en una década se había extendido a otras áreas, incluyendo psiquiatría, cirugía cardiaca, nefrología y neurología. Especialmente esta última área se perfilaba como un ejemplo a nivel nacional (MINSAL, 2015, 2016, 2017). Todo ello justificó la selección de esta unidad como caso para un estudio que, parafraseando a Thomas P. Hughes (2013, p. 101), busca comprender cómo la telemedicina se construye socialmente y configura la sociedad en Chile.

Detrás de estas cifras se escondía una realidad social distinta y definitivamente más compleja. Cuando iniciamos nuestro trabajo de campo en Talcahuano, un fuerte 
conflicto - no relacionado directamente con las operaciones de la Unidad de Teleprocesos (Parra, 2018) - había llevado a la desvinculación del doctor Albornoz. Por el mismo motivo, la directora interina de la Unidad de Hemodinamia y Electrofisiología, que alojaba el programa de telecardiología, declinó participar en el estudio. Perplejo, el jefe de la unidad de Teleprocesos nos contactó con el jefe de la Unidad de Neurología, quien hizo posible que centráramos nuestro estudio en el programa de teleneurología, que consiste en la presentación de casos clínicos en tiempo real con la interacción de médicos neurólogos del Hospital Las Higueras y equipos de Centros de Salud Familiar y otros hospitales de la zona, que no cuentan con especialistas en el área. Pronto fue claro que las noticias que publicitaban la reducción de las listas de espera de especialidad contaban sólo una parte de esta realidad social. La telemedicina se sostiene en un precario equilibrio, apoyada por silenciosas prácticas de mantenimiento y complejas redes de cooperación, competencia y conflicto.

Este artículo es un intento por dar sentido a nuestra experiencia y a la información que pudimos recabar en nuestro trabajo de campo sobre las experiencias de múltiples actores involucrados en la operación rutinaria de la Unidad de Teleprocesos del Hospital Las Higueras, y especialmente en el programa de teleneurología. En particular, este artículo busca utilizar este caso para comprender las tensiones que emergen con la implementación de tecnologías de salud digital y telemedicina. Para ello adopta un enfoque pragmático informado por la semiótica material (Akrich \& Latour, 1992; Callon, 2012), la teoría de la subjetivación (Rose, 1998), y la sociología de la moralidad (Boltanski \& Thévenot, 2006), que permite sacar a la luz el trabajo moral implicado en la realización de medicina a distancia.

En específico, en lugar de evaluar un programa de teleneurología (Patel, Malik, DeMasi, Lunagariya, \& Jani, 2019), este artículo busca dar cuenta de las lógicas que otorgan sentido a cómo en este caso el programa es evaluado por personas que 
participan en él desempeñando distintos roles. Destacaremos tres lógicas que articulan este trabajo moral de la medicina a distancia: una lógica cívico industrial que fundamenta el valor del programa en su contribución a disminuir las listas de espera para acceder a atención en salud; una lógica de la seguridad que fundamenta su valor en la implementación de protocolos válidos para la comunidad profesional; y una lógica del cuidado que lo hace en referencia a la capacidad que tienen los equipos de salud de atender a la situación particular que experimenta cada paciente en esta nueva configuración sociotécnica. Proponemos que reconocer las lógicas que los distintos actores puedan adoptar para otorgar valor a las prácticas de cuidado de salud puede favorecer la comprensión, anticipación y resolución de las tensiones que emergen con la implementación de tecnologías de salud digital y telemedicina.

\section{El análisis de la telemedicina como entramado sociotécnico}

Diversos estudios han enfatizado el carácter vulnerable e inestable de los programas de telemedicina. Desde sus inicios su implementación ha sido difícil y controversial (Sood et al., 2007; Wootton, 1996). Enfrentan desafíos particularmente importantes en regiones donde hay organizaciones gubernamentales débiles y donde no se ha instalado la infraestructura hospitalaria y de telecomunicaciones que los sistemas de telemedicina presuponen. Pero incluso en países con un nivel de desarrollo industrial y económico avanzado los programas de telemedicina no logran superar del todo un estado de alta vulnerabilidad, experimentando dificultades para atraer apoyo institucional y financiero, independizarse de promotores claves, estabilizar un marco regulatorio y financiero, y atraer mayor demanda (OMS, 2010).

A estas dificultades se suma una creciente evidencia de efectos no anticipados de estas tecnologías en diversos ámbitos de la vida social. Una creciente literatura en sociología de la medicina y estudios críticos de la salud digital sugiere que ellas modifican el quehacer cotidiano con objetos físicos y artefactos tecnológicos, 
definiciones de roles, tejidos organizacionales y las relaciones que los pacientes establecen con sus propios cuerpos (Finch, Mort, Mair, \& May, 2008; López Gómez, Callén, Domènech, \& Tirado, 2010; Lupton, 2017; Oudshoorn, 2016)

Frente a estas dificultades, una línea de estudios ha buscado evaluar programas de teleneurología o determinar el nivel de satisfacción de los usuarios (Patel et al., 2019). En este artículo, perseguimos un objetivo complementario: identificar las lógicas que otorgan sentido a cómo un programa de teleneurología es evaluado por personas que participan en él desempeñando distintos roles. De esta manera, este artículo contribuye a la comprensión de las organizaciones de cuidado de salud como contextos sociales polifónicos o policontexturales (Knudsen \& Vogd, 2015), donde se articulan lógicas diversas.

El análisis semiótico material, o teoría del actor-red, desarrollado en el área de los estudios sociales de ciencia y tecnología, constituye una alternativa adecuada para describir y analizar escenarios vulnerables e inestables como éste. Desde esta perspectiva, programas de telemedicina y telecuidado han sido analizados en términos de redes de actores heterogéneos (López Gómez et al., 2010). Se entiende por actor todo ente que hace una diferencia en las redes en que participa, cada uno constituyendo a su vez una red de actores-redes (Callon, 2012). Los entramados sociomateriales incluyen actores de muy diverso tipo: actores humanos y no humanos, incluyendo discursos o instrucciones sobre el modo de operación de las mismas redes (Latour, 2005). De ahí que, en referencia a este programa de investigación, sea apropiado hablar de una semiótica material (Akrich \& Latour, 1992) que permite echar luz sobre los entramados sociomateriales implicados en la realización de medicina a distancia.

Ahora bien, en el análisis del programa de teleneurología nos interesa enfatizar la capacidad crítica de los actores humanos involucrados en estas redes. Para ello nos serviremos de la noción de tecnología humana, propuesta por Nikolas Rose en 
conexión con el trabajo de Foucault sobre las tecnologías del yo. Rose ha definido las tecnologías humanas como ensamblajes híbridos que, junto a personas, edificios y espacios, incluyen sistemas de juicio y se sostienen en “...presupuestos y objetivos acerca de seres humanos" (Rose, 1998, p. 26). En estas perspectivas, la noción de tecnología refiere a más que los artefactos materiales involucrados en el cuidado de la salud: como en la obra de Foucault, técnicas de interacción humana - como la confesión y su heredera, la consulta clínica - constituyen tecnologías. Son humanas en la medida que "...asumen particulares modos de ser humano como su objeto" (1998, p. 26), teniendo como efecto determinadas formas de subjetivación.

Entender los programas de telemedicina, y particularmente un programa de teleneurología, como tecnologías humanas permite enfatizar la capacidad crítica de los actores involucrados en su implementación y operación rutinaria, en términos de trabajo moral. En su trabajo clásico sobre la organización social del trabajo en salud, Strauss, Fagerhaugh, Suczek, \& Wiener (1997) propusieron que éste contempla distintos subtipos de trabajo: de máquinas, seguridad, comodidad, sentimental, articulación y pacientes. Aquí nos referiremos al trabajo moral como aquel en que las personas activan sistemas de juicio para evaluar o legitimar las reconfiguraciones que sufren los ensamblajes sociomateriales en que participan, particularmente de cuidado de salud.

La capacidad crítica desplegada por personas en escenarios tanto públicos como cotidianos también ha sido el foco de la sociología pragmática de la moralidad desarrollada principalmente por Boltanski y Thévenot (2006). En su trabajo sobre los regímenes de justificación, estos autores han dado cuenta de cómo, en situaciones en que el juicio es sometido a una crítica pública, las personas pueden "buscar apoyo sobre ciertos regímenes de justificación que se encuentran ligados a principios que tienen una validez intrínsecamente reconocida" (Boltanski, 2017, p. 179). Estos regímenes constituyen ciudades que entrañan la perspectiva de diseñar un orden 
legítimo a la luz de los principios a los que están ligados. Asimismo, corresponden a distintos órdenes de grandeza, pues permiten establecer el valor de las personas.

Para analizar el despliegue de la capacidad crítica tanto en discursos públicos como en situaciones cotidianas, ampliaremos el análisis de regímenes de justificación al de lógicas, en el sentido propuesto por Annemarie Mol: racionalidades que dan sentido a prácticas situadas. Una lógica determina "lo que es apropiado o lógico hacer en un sitio o situación, y lo que no lo es" (Mol, 2008, p. 10). Desde esta perspectiva, nos centraremos en lógicas que dan sentido a prácticas de valuación y justificación de entramados sociotécnicos.

En definitiva, considerando la implementación y operación rutinaria del programa de teleneurología del Hospital Las Higueras, nos interrogamos sobre ¿qué lógicas otorgan sentido al trabajo moral realizado por personas que ocupan distintos roles en entramados sociotécnicos de teleneurología?

\section{Metodología}

Este artículo se basa principalmente en entrevistas y observaciones no participantes realizadas entre junio y octubre de 2017 en dos hospitales y un Centro de Salud Familiar (CESFAM) del Servicio de Salud Talcahuano, región de Biobío, Chile. Este trabajo fue realizado en el contexto de un estudio más amplio que tiene por objetivo explorar cómo la telemedicina se construye socialmente y configura la sociedad en Chile (Fondecyt No. 11150918). En este estudio hemos entrevistado y observado las prácticas de distintos actores involucrados en la implementación de dos programas de telemedicina.

Concretamente, aquí nos referiremos a uno de los dos casos estudiados: el programa de teleneurología implementado desde 2015 en el Servicio de Salud Talcahuano, coordinado por la Unidad de Teleprocesos del Hospital Las Higueras. Para explorar este caso, en el trabajo de campo enrolamos a un total de 23 personas en tres centros 
de salud que participan en el programa: las Unidades de Teleprocesos y Neurología del Hospital Las Higueras, el Centro de Salud Familiar Paulina Avendaño y el Hospital Penco-Lirquén. El protocolo de investigación utilizado fue aprobado por el Comité Ético Científico de la Universidad Andrés Bello y el del Servicio de Salud Talcahuano, contemplando el consentimiento informado de todos los participantes.

Las 23 personas enroladas en esta fase del estudio fueron seleccionadas por ocupar distintas posiciones y roles en el programa de teleneurología en estos tres centros: ocho médicos de atención primaria y neurólogos involucrados en su implementación en cada tipo de centro de salud; ingenieros responsables de la administración y el mantenimiento del sistema; personal de la Unidad de Teleprocesos del hospital de alta complejidad; personal directivo del CESFAM; y nueve pacientes del CESFAM que han sido usuarios de este programa.

Varios participantes fueron seleccionados porque eran los únicos ocupantes de una posición en el programa. En el caso de los médicos de atención primaria y neurólogos en los hospitales, se procedió según un criterio de conveniencia: cumpliendo la condición de tener experiencia en la implementación del programa de teleneurología, se seleccionó a quienes estuvieron disponibles para entrevistarse con nosotros en alguna de las dos visitas que realizamos a estos centros de salud, las cuales tuvieron una duración de una semana, respectivamente. Entrevistamos a cada uno de estos actores en al menos una ocasión. Realizamos entrevistas de seguimiento a quienes lideran la implementación del programa para conocer avances y profundizar en aspectos de relevancia. En total se realizaron 18 entrevistas semiestructuradas a miembros de los equipos de salud con una duración aproximada de 90 minutos cada una.

Por su parte, los pacientes fueron reclutados también según un criterio de conveniencia. Ocho fueron alistados durante una mañana en la consulta de teleneurología: su consulta ya había sido agendada para ese día por la médica de 
atención primaria del CESFAM según el procedimiento de rutina. Todos presentaban condiciones de salud distintas. A cada paciente se le presentaron los objetivos y las condiciones del estudio, y se le pidió su consentimiento para que el investigador responsable estuviera presente durante la consulta y pudiéramos realizarle una entrevista con posterioridad. Una paciente no quiso atenderse en modalidad de teleneurología en esa ocasión, pero accedió a entrevistarse con nosotros para dar a conocer sus razones. Observamos presencialmente la participación de siete pacientes en consultas de teleneurología. Dos de los pacientes observados estuvieron disponibles con posterioridad para entrevistarse con nosotros. Una última paciente fue enrolada en la Unidad de Teleprocesos del Hospital Las Higueras cuando asistía a retirar órdenes médicas después de recibir atención por teleneurología. En total se realizaron entrevistas semiestructuradas a cuatro pacientes, con una duración que varió considerablemente entre los 30 minutos y los 140 minutos.

\section{Las lógicas del trabajo moral en teleneurología}

\section{La lógica de la eficiencia cívica}

Al ingresar al Hospital Las Higueras de Talcahuano el primer día de nuestro trabajo de campo, en junio de 2017, notamos la fotocopia de una noticia, presumiblemente de un periódico local, pegada en una ventana. El encabezado leía, "Disminuyen lista de espera y mortalidad en pacientes de neurología". En el texto se destacaba la incidencia del programa de telemedicina:

Para el médico, gran parte del éxito se debe también al trabajo de la unidad en el Programa Telemédico del Hospital Las Higueras. Desde 2015 a la fecha, se han atendido en esta modalidad poco más de mil 370 usuarios con mil 704 Teletransacciones. 
En estos espacios públicos - un periódico y el vestíbulo del Hospital - la variación en la lista de espera actuaba como prueba del valor de una manera de configurar el cuidado de salud, que contempla la introducción de tecnologías de telemedicina. Los estudios de Boltanski y Thévenot (2006) sobre los regímenes de justificación, y especialmente su análisis de cómo se establecen compromisos entre el régimen cívico y el industrial, permiten comprender la posición privilegiada que ha llegado a ocupar la lista de espera en este sitio.

Boltanski y Thévenot (2006) han argumentado que, en situaciones donde se deben dar razones de manera pública, las personas buscan apoyo en regímenes de justificación. Los regímenes del mundo cívico y del industrial son de especial relevancia para comprender cómo se pone en valor el programa de teleneurología en discursos públicos. El régimen del mundo cívico se caracteriza por referir a los colectivos que las personas constituyen al reunirse, y donde acceden a estados de valor. En referencia a este mundo, las acciones de quienes están involucrados en la implementación de programas de telemedicina deben ser justificables atendiendo al derecho que tiene cada persona en cuanto perteneciente al colectivo.

Por su parte, el régimen de justificación del mundo industrial otorga valor a las personas según pruebas de eficiencia y productividad, que se expresan en medidas y estadísticas (Boltanski \& Thévenot, 2006). Aquí, quien se involucra en la implementación de programas de telemedicina ha de justificar su acción en términos de un aumento de la eficiencia del sistema de salud.

La sistemática referencia a la lista de espera como prueba de valor delata un compromiso entre ambos mundos, como el que Boltanski y Thévenot reconocieran en su análisis de servicios públicos en general: “...medidas que tienen la intención de aumentar la eficiencia son justificadas, especialmente por los miembros del servicio, por una preocupación por el bien común de los usuarios" (Boltanski \& Thévenot, 2006, p. 331). En este compromiso cívico industrial, un programa de telemedicina constituye 
un entramado sociotécnico que, en referencia al principio de legitimidad industrial, debe proveer un aumento en la productividad del sistema de salud. Ahora bien, esto mismo sólo puede ser justificado por alusión a "un conjunto de textos y reglas que encarnan la voluntad general" (Boltanski \& Thévenot, 2006, p. 331); es decir, por referencia a los derechos de las personas que componen colectivos.

A ello llamaremos una lógica de la eficiencia cívica. En este contexto, la lista de espera constituye un objeto peculiar como medida de eficiencia de los entramados sociotécnicos y prueba de satisfacción de la voluntad general, según ésta se expresa en un cuerpo legal.

En el caso del programa de teleneurología del Servicio de Salud Talcahuano, la actuación de la lista de espera como prueba de valor es particularmente clara en una serie de publicaciones en medios de comunicación de masas, que replican la que encontráramos publicada en el vestíbulo del hospital. Así, cuando el Ministerio de Salud del Gobierno de Chile anunció la pronta inauguración de este programa en 2015, señaló que: "Lo que nosotros pretendemos es agilizar estos procesos de manera de reducir la mortalidad y la morbilidad y llevar la lista de espera a cero" (MINSAL, 2015). En años subsiguientes se comunicaban sus frutos: "Programa de Telemedicina del Hospital Las Higueras logra disminución de listas de espera" (MINSAL, 2016); “...uno de los grandes logros de este año de labor ha sido la disminución de la lista de espera de Neurología Adulto" (MINSAL, 2017). Esta forma de valorar y visibilizar al programa de telemedicina pone énfasis en su capacidad resolutiva de demandas colectivas en torno a la oportuna atención en salud.

Como compromiso cívico industrial, esta prueba requiere que se haga alusión a una serie de textos que justifiquen este aumento de eficiencia en alusión a la voluntad general. En este caso, ello se logra por referencia a la Constitución Política de la República, que establece el derecho a la vida e integridad física, y al Decreto Supremo N³ del Ministerio de Salud, que aprueba el Régimen de Garantías en Salud, 
estableciendo tiempos y modalidades de atención para distintas condiciones de salud (Castillo-Sepúlveda et al., 2017).

De esta manera, la reducción de los tiempos de espera de los pacientes, medida en términos de las listas de espera de primera consulta especializada, otorga un indicador concreto del valor del programa, según criterios de eficiencia técnica y de respeto a derechos. La lista de espera actúa como prueba de valor para el compromiso cívico industrial que legitima la implementación del programa de telemedicina.

\section{La lógica de la seguridad}

Pero cuando hablamos con los médicos que participan en el programa de telemedicina, las listas de espera perdieron protagonismo. En su lugar, los médicos se mostraron preocupados de controlar riesgos inherentes a la realización de medicina a distancia. Este no es un problema de eficiencia cívica, sino uno que corresponde a una lógica de la seguridad. Ahora bien, mientras en sus discursos los médicos enfatizan esta lógica, en la práctica atienden simultáneamente a una lógica del cuidado, si bien dicho énfasis difiere entre médicos neurólogos y de atención primaria.

Estudios previos han observado ya que la telemedicina debe responder simultáneamente a las lógicas de la seguridad y del cuidado (López Gómez et al., 2010). En una lógica de la seguridad se enfatiza el control de las variables que inciden en una condición de salud. Para ello las personas deben estar en condiciones de entregar evidencias de que se atienen a estándares, protocolos y guías prácticas clínicas que establecen patrones de acción ideados justamente para reducir el riesgo. En esta lógica, la persona es comprendida como quien toma decisiones y debe hacerse responsable de sus consecuencias, ya sean miembros de equipos de salud o pacientes (Mol, 2008). En una lógica del cuidado, en cambio, el énfasis se vuelca hacia el carácter impredecible de los cuerpos y de la situación de cuidado. Se basa en una sistemática consideración de lo que desborda los protocolos, en el soporte 
emocional de pacientes y sus personas cercanas, y en la atención de los complejos entramados de las situaciones de vida en las que ellos se ven implicados (López Gómez et al., 2010; Mol, 2008).

A la luz de una lógica de la seguridad, el programa de telemedicina requiere que rutinariamente se generen registros que puedan utilizarse como prueba de que las prácticas de atención en teleneurología se atienen a protocolos válidos para la comunidad profesional. Para ello, el programa cuenta con el "Protocolo de Referencia Programa Teleneurología”, fijado por resolución exenta n²91 del Servicio de Salud Talcahuano. Este protocolo, "establece las responsabilidades, alcance y descripción del proceso de atención y contrarreferencia de interconsulta de atención primaria...”. En él se describen los procesos que pueden tener lugar en la consulta virtual sincrónica (tales como atención, citación, solicitud o realización de exámenes, recetas médicas e indicación de hospitalización), los distintos roles que participan ellos y sus responsabilidades, incluyendo procedimientos administrativos y criterios de derivación. A su vez, este protocolo refiere a otros más generales, tales como los de Manejo de Dolor Neuropático o de Cefalea Crónica. Mediante la aplicación de estos protocolos, el sistema de telemedicina al mismo tiempo controla riesgos y distribuye responsabilidades, asegurando trazabilidad.

Aun así, al introducir el programa de neurología algunos médicos neurólogos del servicio de salud se opusieron a participar en él, aduciendo que la interacción a través de pantallas no permitía seguir estándares de atención que son propios de la especialidad. En esta modalidad, el paciente es atendido presencialmente por un médico de atención primaria en una sala del Centro de Salud Familiar, mientras el médico neurólogo se encuentra en la sala de teleprocesos del hospital. Para realizar exámenes neurológicos, este último dialoga con los pacientes y les instruye que realicen distintos movimientos. En todo momento cuenta con el apoyo del médico de atención primaria, quien posiciona y orienta al paciente según las necesidades del 
protocolo de examinación y del equipo de teleconferencia. Ahora bien, algunos exámenes deben ser realizados por el médico de atención primaria, lo que pone a los neurólogos en guardia. Si bien los médicos generales que participan en el programa han recibido entrenamiento para realizar estas maniobras, tanto en el curso de su entrenamiento formal en la escuela de medicina como en su introducción al programa de teleneurología, los neurólogos ven abrirse aquí una ventana de riesgo, que algunos no han estado dispuestos a asumir. En palabras de una médica neuróloga:

Yo encuentro que lo más complicado es que los médicos de los consultorios, aunque se han ido capacitando en el examen neurológico, hay algunas técnicas en el examen que no han podido hacer con mucha certeza. Por ejemplo, fondo de ojo y campos visuales son técnicas que uno hace con más detalle que los médicos en los consultorios.

De acuerdo con una lógica de la seguridad, el médico es responsable de las decisiones que toma y de los riesgos que traen consigo. De ahí que los neurólogos no puedan ser obligados formalmente a participar en el programa de teleneurología, con la consecuencia de que un grupo de especialistas se restara de él.

\section{La lógica del cuidado}

Una paciente nos relató su primera experiencia en el programa de teleneurología del siguiente modo. En la sala de espera del Centro de Salud Familiar, la médica de atención primaria se le acercó para pedirle que pasara a la consulta. Le advirtió que sería una teleconferencia, por lo que ella pensó que se trataría de una conferencia televisada sobre condiciones de salud: "Yo pensé que estaría ella con muchos pacientes más; que les iba a explicar algo de la enfermedad. Yo entre así: oh... [gesto desanimado]. Me van a dar charla, ipero yo quiero que a mí me atiendan!"

Su demanda por atención tiene sentido para las tres lógicas que hemos identificado. Para una lógica de la eficiencia cívica, ella demanda que se respete su derecho a una 
atención oportuna y de calidad. Para una lógica de la seguridad, ella demanda que se le atienda según estándares de calidad de la profesión, asumiendo responsabilidad por las decisiones que tome. Para una lógica del cuidado, demanda que su situación y su experiencia particular sea tenida en consideración.

La necesidad de articular una lógica de la seguridad con una del cuidado obliga al equipo médico a poner atención a lo que sobrepasa el protocolo, llevando a cabo prácticas que continuamente lo ponen a prueba (López Gómez et al., 2010, p. 72). En el programa de teleneurología se produce una división del trabajo de cuidado según tipo de establecimiento de salud, liberando significativamente al médico neurólogo de la responsabilidad de establecer relaciones sociales centradas en la lógica del cuidado, y constituyendo al mismo tiempo a los establecimientos de salud primaria como sitios de proximidad.

Esto no libera al médico neurólogo de la necesidad de establecer estrategias que anticipan la lógica del cuidado. Su articulación con una lógica de la seguridad es explícita en la manera cómo un especialista describe el proceso de atención. Por una parte, atendiendo a una lógica del cuidado, utiliza dramatúrgicamente el medio que tiene a su disposición en la interacción mediada para hacer que los pacientes sientan que están siendo atendidos:

...que sienta que tú la estas envolviendo. ¿Te fijas? Ser empático. Después dices: ‘¡Señora, espéreme un momento!'. Eso es super importante. ‘¡Espéreme un momento, tengo que leer su historia!'. Yo podría haber leído la historia antes. Obvio. Pero eso es una actuación. ¿Te fijas? La persona dice: ¡Oh, el doctor me va a leer mi historia!

Por otra parte, atendiendo a una lógica de la seguridad, verifica la información que se le ha entregado en la ficha clínica, según los protocolos de atención:

Todo eso está escrito, pero tú lo vuelves a preguntar, tú haces un doble chequeo de la información. Nunca la información entregada es verdadera. 
Siempre hay que partirla por dos. Porque el otro es un médico general, y eventualmente esa señora le mintió al doctor, o no se acordaba en entregarle toda su información, o el doctor no tenía experticia para sacarle información.

Si bien la intermediación de pantallas obliga al médico neurólogo a desplegar dichas estrategias de dramatización, al mismo tiempo lo libera de la mayor parte de la carga de cuidado, que recae en el personal de atención primaria. En palabras de un médico de atención primaria, el ambiente hostil del hospital contrasta con la familiaridad del Centro de Salud Familiar: “...el hospital es gigante, es un poco más como hostil, por decirlo así. Es como algo más familiar para la gente el lugar, el personal, la gente... aquí hay gente que trabaja hace mil años. Entonces, los conocen de nombre." De acuerdo con esta división del trabajo, los médicos de atención primaria asumen la mayor carga de cuidado: al finalizar la consulta con el médico neurólogo por teleconferencia, acompañarán a los pacientes, los ayudarán a vestirse y a incorporarse, y se asegurarán de que hayan comprendido las instrucciones que se les hayan dado, orientándolos en las próximas acciones a tomar y construyendo una relación de acompañamiento a largo plazo.

Si bien las pacientes hacen referencia a criterios propios de una lógica de eficiencia cívica al evaluar el programa de teleneurología, señalando que valoran ser atendidas eficientemente de acuerdo con sus derechos, ponen énfasis en criterios propios de una lógica del cuidado, según la cual verifican que su situación particular se hace visible para el sistema administrativo de cuidado de la salud. Alice Street (2014) ha puesto énfasis en esta dimensión de la experiencia de los pacientes: en sus manos, tecnologías como tarjetas de salud y resultados de exámenes son vaciadas de contenido biomédico y pasan a actuar como tecnologías de visibilidad social. Por medio de estos objetos, los pacientes adoptan una forma que es visible en espacios de salud, obligando a sus contrapartes a establecer una relación social con ellos. 
En la atención de teleneurología, las pacientes verifican continuamente si sus cuerpos y sus experiencias particulares se han hecho visibles, destacando cinco aspectos: a) son atendidas por dos médicos simultáneamente, b) que demuestran estar enfocados en la interacción; c) se realizan exámenes físicos, y d) se solicita realizar exámenes en otras dependencias del hospital, e) llevando a la generación de un diagnóstico que da sentido a su experiencia de la enfermedad.

Una paciente resalta que ser atendida por dos médicos simultáneamente equivale a recibir el doble de atención: Me sentí importante. Me sentí privilegiada porque me iban a ver dos médicos a la vez. Yo [...] pensé que la doctora se iba a ir. Pero la doctora se quedó, cerró la puerta y listo". Esto es recalcado en comparación con el tipo de atención disponible en proveedores privados, a los que sistemáticamente las entrevistadas atribuyen mejores condiciones: "...te digo de verdad, ni particular la habría tenido. Ni particular. Para mí, algo extraordinario, porque en ese instante me estaban atendiendo dos médicos; dos médicos. Una me está examinando y el otro está dando la opinión como especialista."

Pese a que el médico neurólogo no está presente en la consulta, pacientes expresan haber sentido que él estaba atento a su experiencia particular: “...el especialista (...) tú no lo ves. O sea, no: no lo tocas, nada. Pero el médico, al fondo, está en un cien por ciento sobre ti.” Lo mismo recalca otra paciente: “...a mí me encantó esto, porque está el doctor, está el mismo doctor viendo. Y le decía a la señorita lo que tenía que hacer. La realización de exámenes en otras dependencias es experimentada también como una forma de adquirir visibilidad. Esto es particularmente significativo cuando estos pacientes no han tenido acceso a este modo de aparecer previamente: "Primera vez, imagínate, primera vez en mi vida que un médico [...] me manda a hacer un scanner a la columna. O sea, encuentro... es lo máximo para mí." La tecnología de imagenología médica actúa como una tecnología de visibilidad social (Street, 2014). Ella fuerza a los miembros de organizaciones de cuidado de salud a establecer una 
relación social en que su situación particular es atendida y, en último término, nombrada: “...quería darle las gracias, porque [...] usted me está dando la respuesta de por qué tengo esta enfermedad".

Así, la consulta teleneurológica es validada por las pacientes cuando comprueban que las ha hecho visibles en el espacio biomédico. Sus experiencias son atendidas cercanamente por dos médicos que colaboran en la realización de exámenes en el espacio de interacción presencial, solicitan la realización de exámenes que requieren máquinas especializadas, y generan diagnósticos que nombran y articulan las experiencias de la enfermedad bajo nuevos parámetros de normalidad.

\section{Telemedicina, controversia y cambio social}

Tres lógicas dan sentido simultáneamente a prácticas de valuación del programa de teleneurología desplegadas por actores que desempeñan roles distintos, generando puntos de tensión y encauzando la constante evolución de los entramados sociomateriales en que se realiza el cuidado de salud.

Desde una lógica de eficiencia cívica, que da cuenta de un compromiso cívico industrial (Boltanski \& Thévenot, 2006), las autoridades del servicio de salud dan a conocer los resultados de su gestión en términos de la reducción en las listas de espera de especialidad. Cuando la reducción es significativa, autoridades en distintos niveles adquieren, en medios de comunicación de masas, la forma de héroes por haber mejorado la capacidad del sistema para actuar en pos del bien común de los ciudadanos.

Miembros del personal médico especializado, en tanto, consideran desde una lógica de la seguridad que esta presión por mejorar la eficiencia del sistema implica un aumento del riesgo que deben asumir, llegando algunos de ellos a marginarse de su operación. Para ellos, las fotografías que las autoridades políticas y administrativas se toman junto a las máquinas de telemedicina son fatuas, aun cuando comprendan la 
lógica que las anima. Resienten al mismo tiempo que, para la realización de exámenes, dependan de los ojos y las manos de médicos generales con menor entrenamiento y prestigio. Para quienes privilegian criterios de eficiencia cívica, estas quejas delatan más bien que los médicos especialistas han sido heridos en su orgullo. A la inversa, si bien médicos de atención primaria valoran las posibilidades de aprendizaje que la teleneurología abre al permitirles interactuar sistemáticamente con especialistas, resienten una falta de reconocimiento a su capacidad de realizar adecuadamente maniobras para las que han sido entrenados. Igualmente, sienten invisibilizadas sus prácticas de cuidado: la energía y el tiempo que invierten en prácticas rutinarias de atención y contención emocional, que se suman a tareas de mantenimiento como la selección y citación de los pacientes que serán atendidos por teleneurología.

Pacientes, en tanto, claman por ser atendidos con oportunidad, calidad y dignidad. Si bien toman responsabilidad por su situación de salud en la medida de sus medios, no entienden del todo las alternativas que tienen a su disposición. No es una lógica de la seguridad o la elección la que privilegian, sino del cuidado (López Gómez et al., 2010; Mol, 2008). Utilizan los medios que tienen a su disposición como tecnologías de visibilidad social para que sus cuerpos y sus experiencias individuales se hagan visibles en sitios biomédicos y que sus dolencias sean nombradas (Street, 2014). Para los pacientes, la lista de espera de especialidad adquiere la forma de un túnel oscuro que impide que la mirada clínica se pose en ellos y les ayude a dar sentido a sus experiencias. Esto explica la reticencia de quienes temen que la intermediación de pantallas no sea sino otra forma de ausencia. $\mathrm{Y}$ asimismo explica el alivio de que quienes experimentan que, a pesar de estar frente a pantallas, son acompañados y atendidos con cuidado.

Por lo mismo, los pacientes resienten cuando prima cualquiera de las otras lógicas, como puede ocurrir en la somatocracia del Régimen de Garantías Explícitas en Salud 
(Castillo-Sepúlveda et al., 2017). Por una parte, cuando prima la referencia a garantías de atención en salud que otorga o resta prioridad a los pacientes según criterios de eficiencia cívica que, para ellos, permanecen opacos y desconectados de su experiencia concreta de la enfermedad. Por otra parte, cuando las relaciones sociales se atienen ciegamente a protocolos de seguridad en la atención que, para ellos mismos, son tan fríos como irrelevantes.

Estas lógicas de la eficiencia cívica, la seguridad y el cuidado, encauzan procesos de cambio social, en la forma de una evolución de los entramados sociomateriales implicados en el cuidado de salud. En cada sitio y momento podrán privilegiarse unas sobre otras, derivando en entramados que desembocan en controversias o conflictos: es decir, en disputas en torno a la legitimidad de entramados sociotécnicos en salud digital. Por ello proponemos que reconocer y anticipar las lógicas que los distintos actores puedan adoptar para otorgar valor a las prácticas de cuidado de salud, puede favorecer la comprensión, anticipación y resolución de las tensiones que emergen con la implementación de tecnologías de salud digital y telemedicina. Esto requiere atender a las distintas lógicas que dan sentido al trabajo moral en telemedicina.

\section{Agradecimientos}

Esta publicación es resultado del proyecto CONICYT Fondecyt Iniciación en la Investigación Folio 11150918, "Telemedicina, controversia y cambio social en Chile: cómo la telemedicina se construye socialmente y configura la sociedad". Agradecemos a todos quienes participaron e hicieron posible este estudio: a los pacientes y miembros de equipos de cuidado de salud que accedieron a relatar sus experiencias; al jefe de la Unidad de Teleprocesos del Hospital Las Higueras, Gerardo Vergara, y al jefe de la Unidad de Neurología, Dr. Freddy Constanzo; y a Catalina Silva, que participó en calidad de asistente de investigación en la recolección de datos y en su sistematización inicial. 
Agradecemos las observaciones y sugerencias realizadas por dos revisores anónimos, que permitieron hacer mejoras significativas a este trabajo.

\section{Referencias}

Akrich, M., \& Latour, B. (1992). A summary of a convenient vocabulary for the semiotics of human and nonhuman assemblies. En W. E. Bijker \& J. Law (Eds.), Shaping technology/Building society. Studies in sociotechnical change (pp. 259-264). Cambridge, Mass. - London, England: The MIT Press.

Boltanski, L. (2017). Un nuevo régimen de justificación: La ciudad por proyecto. Entramados y Perspectivas, 7(7), 179-209.

Boltanski, L., \& Thévenot, L. (2006). On Justification: Economies of Worth. Princeton University Press.

Callon, M. (2012). Society in the Making: The Study of Technology as a Tool for Sociological Analysis. En W. E. Bijker, T. P. Hughes, \& T. Pinch (Eds.), The social construction of technological systems: New directions in the sociology and history of technology (Anniversary Edition, pp. 77-97). Cambridge, Mass.: The MIT Press.

Carvajal, Y., González, I., \& Pacheco, J. (2013). Francisco Albornoz o moviendo la prodigiosa red pública en las proximidades de C. Rev Chil Salud Pública, 17(2), $147-150$.

Castillo-Sepúlveda, J., Espejo, M., Tapia, J., Catalán, M., Toro, J., \& Gálvez, M. (2017). Tecnologías, episteme y subjetivación en un régimen de garantías en salud. Psicoperspectivas. Individuo y Sociedad, 16(3), 6-16.

Finch, T. L., Mort, M., Mair, F. S., \& May, C. R. (2008). Future patients? Telehealthcare, roles and responsibilities. Health \& Social Care in the Community, 16(1), 86-95. https://doi.org/10.1111/j.1365-2524.2007.00726.x 
Hughes, T. P. (2013). La Evolución de los Grandes Sistemas Tecnológicos. En A. Buch \& H. Thomas (Eds.), Actos, actores y artefactos (pp. 101-145). Bernal: Universidad Nacional de Quilmes Editorial.

Knudsen, M., \& Vogd, K. (Eds.). (2015). Systems theory and the sociology of health and illness: Observing healthcare. London: Routledge.

Latour, B. (2005). Reassembling the Social: An Introduction to Actor-Network-Theory. New York: Oxford University Press.

López Gómez, D., Callén, B., Domènech, M., \& Tirado, F. (2010). How to become a Guardian Angel. Providing safety in a Home Telecare Service. En A. Mol, I. Moser, \& J. Pols (Eds.), Care in Practice. On Tinkering in Clinics, Homes and Farms (pp. 71-90). Bielefeld: Transcript Verlag.

Lupton, D. (2017). Digital Health (Critical Approaches to Health). Routledge.

MINSAL. (2015, septiembre 4). Hospital Las Higueras de Talcahuano: Importantes adelantos permiten reducir listas de espera. Recuperado de Noticias Regionales website: https://www.minsal.cl/1022/

MINSAL. (2016, enero 18). Programa de Telemedicina del Hospital Las Higueras logra disminución de listas de espera. Recuperado de Noticias Regionales website: https://www.minsal.cl/disminucion-de-listas-de-espera-logra-programa-detelemedicina-del-hospital-las-higueras/

MINSAL. (2017, abril 12). Unidad de Neurología Adultos de Las Higueras interviene exitosamente lista de espera de la especialidad. Recuperado de Noticias Regionales website: https://www.minsal.cl/unida-de-neurologia-adultos-de-lashigueras-interviene-exitosamente-lista-de-espera-de-la-especialidad/

Mol, A. (2008). The Logic of Care: Health and the problem of patient choice. London and New York: Routledge. 
OMS (Ed.). (2010). Telemedicine: Opportunities and developments in member states: report on the second Global survey on eHealth. Geneva, Switzerland: Organización Mundial de la Salud.

Oudshoorn, N. (2016). Telecare technologies and the transformation of healthcare. London: Palgrave Macmillan.

Parra, N. (2018, noviembre 12). Médico en la mira: Negaría atención a pacientes en hospital para derivarlos a clínica privada. biobiochile.cl. Recuperado de https://www.biobiochile.cl/noticias/nacional/region-del-biobio/2018/11/12/medico-en-la-mira-negaria-atencion-a-pacientes-en-hospitalpara-derivarlos-a-clinica-privada.shtml

Patel, U. K., Malik, P., DeMasi, M., Lunagariya, A., \& Jani, V. B. (2019). Multidisciplinary Approach and Outcomes of Tele-neurology: A Review. Cureus, 11(4). https://doi.org/10.7759/cureus.4410

Rose, N. (1998). Inventing Our Selves: Psychology, Power, and Personhood. Cambridge - New York - Melbourne: Cambridge University Press.

Sood, S., Mbarika, V., Jugoo, S., Dookhy, R., Doarn, C. R., Prakash, N., \& Merrell, R. C. (2007). What Is Telemedicine? A Collection of 104 Peer-Reviewed Perspectives and Theoretical Underpinnings. Telemedicine and e-Health, 13(5), 573-590. https://doi.org/10.1089/tmj.2006.0073

Strauss, A. L., Fagerhaugh, S., Suczek, B., \& Wiener, C. L. (1997). Social Organization of Medical Work. [Nueva Jersey]: Transaction Publishers.

Street, A. (2014). Biomedicine in an Unstable Place: Infrastructure and Personhood in a Papua New Guinean Hospital. Durham: Duke University Press Books.

Wootton, R. (1996). Telemedicine: A cautious welcome. BMJ : British Medical Journal, 313(7069), 1375-1377. 


\section{Declaración de contribución de autores}

FV es investigador principal de este estudio, siendo responsable del diseño, implementación y análisis de resultados. NS contribuyó a su implementación y análisis de resultados en calidad de asistente de investigación. Ambos autores discutieron los resultados y contribuyeron a la versión final del manuscrito.

\section{Formato de citación}

Valenzuela, F. y Sibrian, N. (2019). Las Lógicas del Trabajo Moral en Telemedicina: Disputas en Torno a la Legitimidad de Políticas de Salud Digital. Psicología, Conocimiento y Sociedad, 9(2), 273-297. doi: http://dx.doi.org/10.26864/PCS.v9.n2.11 